\title{
Duodenal angiodysplasia: a case report
}

\author{
Tamer A. Ali ${ }^{1 *}$ (D) Wael Ibrahim², Mohamed A. Tawab ${ }^{3}$ and Mona Abdel Ghaffar ElHariri ${ }^{4}$
}

\begin{abstract}
Background: Angiodysplasia (AD) is an abnormal, ectatic dilated, tortuous blood vessel that is found in the mucosa and the submucosa of the gastro-intestinal tract (GIT). While colonic angiodysplasia is a recognized finding of the lower intestinal tract in the elderly, small intestinal angiodysplasia is rare. However, it is an important reason of GIT bleeding so its detection and proper management can be a challenge. It should be considered among the differential diagnosis in the scenario of mild or intermittent GIT bleedings of obscure cause.

Case presentation: A 71-year-old woman was presented to our emergency department with hypovolemic shock due to lower GIT bleeding, and she was suffering of melena and severe anemia. The revision of past medical history revealed a history of hypertension, diabetes mellitus, and chronic renal disease. After stabilization, she underwent abdominal computed tomography (CT) which revealed a small abnormal vascular lesion along the anterior and posterior wall of the 2nd part of the duodenum. It appeared as blush of contrast in the arterial phase (representing dilated mucosal capillaries draining into tortuous submucosal vein) suggestive of vascular lesion (duodenal angiodysplasia). The patient was transferred to undergo an angiogram which confirmed the diagnosis of duodenal angiodysplasia. Super selective cannulation of the feeding artery was performed followed by post coiling angiogram which revealed successful embolization. No acute complications were encountered during or immediately after procedure.

Conclusion: $A D$ is a rare but important cause that should be considered in the differential diagnosis of GIT bleeding especially in the older patients. It should be looked for in CT angiography done in such a clinical situation. Superselective coil embolization is a safe and effective technique to manage bowel AD.
\end{abstract}

Keywords: Angiodysplasia, Gastrointestinal hemorrhage, Selective embolization, Interventional procedure

\section{Background}

$\mathrm{AD}$ is an abnormal, ectatic dilated, tortuous blood vessel that is found in the mucosa and the submucosa of the GIT. It is usually sub-centimeter in its diameter. While the first case of angiodysplasia (AD) was described in the literature in 1839, that term (AD) was first defined in 1974 as abnormal mucosal vessel clusters in the colon. There was a debate regarding its true etiology that had led to having multiple synonymous terms such as angioectasia, arteriovenous malformations (AVM), and vascular ectasia [1-4].

\footnotetext{
* Correspondence: Drtamerfathi@yahoo.com

'Department of Radio-diagnosis, Faculty of Medicine, Zagazig University, PO BOX 184, Sharkia, Zagazig 44511, Egypt

Full list of author information is available at the end of the article
}

The prevalence of angiodysplasia is increased in older populations, in patients with GI bleeding, and in patients with certain predisposing conditions such as end-stage kidney disease, von Willebrand disease, and possibly aortic stenosis. While colonic angiodysplasia is a recognized finding of the lower intestinal tract in the elderly, small intestinal angiodysplasia is rare. $\mathrm{AD}$ of the stomach or duodenum has been assumed as the reason of blood loss in 4 to $7 \%$ of patients with GI bleeding [5-7]. AD is usually detected at endoscopy being done to evaluate gastrointestinal (GI) bleeding, but in some cases, radiographic imaging or surgery may be required for detection [6-9]. So, detection and proper management of AD can be a challenge and should be considered among the differential diagnosis in the scenario of mild or intermittent GIT bleedings of obscure cause [3, 5-9]. 
a

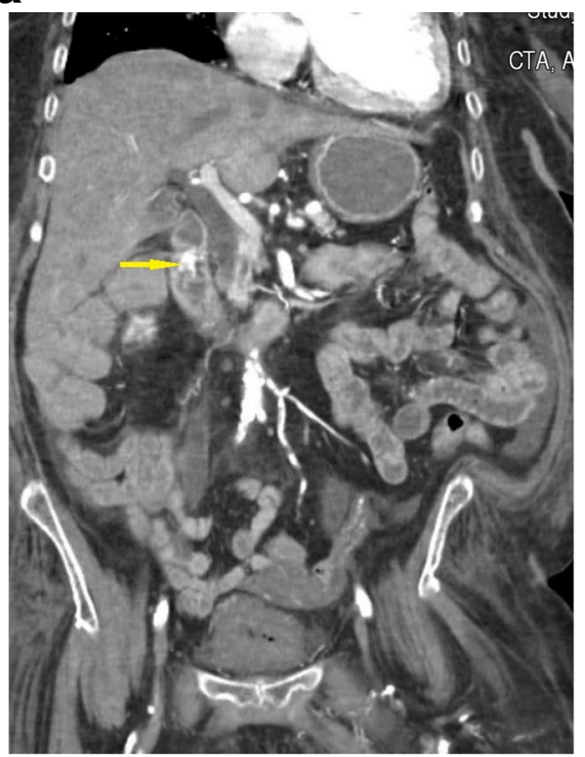

b

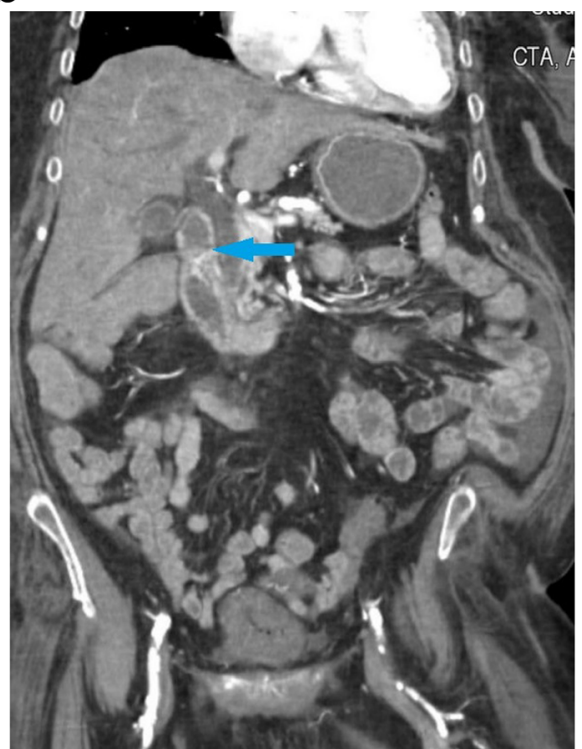

Fig. 1 a, b CT coronal reconstruction showing a small vascular lesion (yellow arrow) at 2nd part of the duodenum with a blush of contrast- and tortuous-related vessels (blue arrow) suggesting duodenal angiodysplasia

\section{Case presentation}

A 71-year-old woman was presented to our emergency department with hypovolemic shock due to lower GIT bleeding. She was suffering of melena and severe anemia. The revision of past medical history revealed a history of hypertension, diabetes mellitus, and chronic renal disease. The clinical examination showed a blood pressure of $90 / 50 \mathrm{mmHg}$, adequate bilateral air entry, and soft abdomen. Laboratory tests showed low hemoglobin (8 g/ $\mathrm{dL})$ and impaired renal function. After stabilization by packed red cell transfusion, she underwent abdominal ultrasound which apart from the small kidneys was unremarkable. CT was done using Siemen (Somatom definition edge) 128 slices machine. The CT examination started by a preliminary unenhanced low-radiation dose series, then injection of intravenous contrast material through an antecubital vein with (total dose of $100 \mathrm{~mL}$ at rate of $4 \mathrm{~mL} / \mathrm{s}$ ), followed by a bolus of a $50-\mathrm{mL}$ saline. The arterial phase images were done using automated bolus triggering (started when the attenuation coefficient in the proximal abdominal aorta reaches $150 \mathrm{HU}$ ). Portal venous phase was obtained 1 -min later.

CT (Fig. 1) revealed a $1.2 \times 1.5 \mathrm{~cm}$ abnormal small vascular lesion along the anterior and posterior wall of the 2nd part of the duodenum which appeared as blush of contrast in the arterial phase (representing dilated mucosal capillaries draining into tortuous submucosal vein) consistent with vascular lesion (duodenal angiodysplasia).

After informed consent, the patient was transferred to undergo angiogram (Fig. 2). Under complete aseptic technique and local anesthesia, a right common femoral artery access was secured by $5 \mathrm{~F}$ sheath, through which 5F Sim II was manipulated over guide wire and placed at proximal part of the abdominal aorta (just above celiac trunk origin). Abdominal aortogram was done which showed the celiac trunk at T12. The annulation of the celiac artery followed by digital subtraction angiography (DSA) revealed celiac branches, coaxial selective cannulation of gastroduodenal artery showed abnormal arterial extravasation at the same area seen CT (suspected angiodysplasia). Super selective cannulation of feeding artery using Codman 21 microcatheter over Agility micro wire (ASAHI INTECC USA, Inc.) was done. That was followed by coil embolization using (4 pieces: $10 \mathrm{~mm}$ in the proximal part and others $5 \mathrm{~mm} \times 30 \mathrm{~cm}$ Concerto Helix coils, EV3 midtronic) from distal to proximal. The aim was trapping of the lesion to prevent backflow. No other embolic agent was used. Post coiling angiogram was done reveal no more active arterial extravasation. Additionally, selective cannulation and DSA of the superior mesenteric artery (SMA) were done to be sure no retrograde filling of the lesion and to assess opened anastomosis which fills the branches of GDA branches. It revealed normal branches with no active extravasation. Femoral access sheath was removed, and hemostasis was secured by manual compression. No acute complications were encountered during or immediately after procedure. Clinically, improvement of the patient was achieved and no further complication was reported in 6 


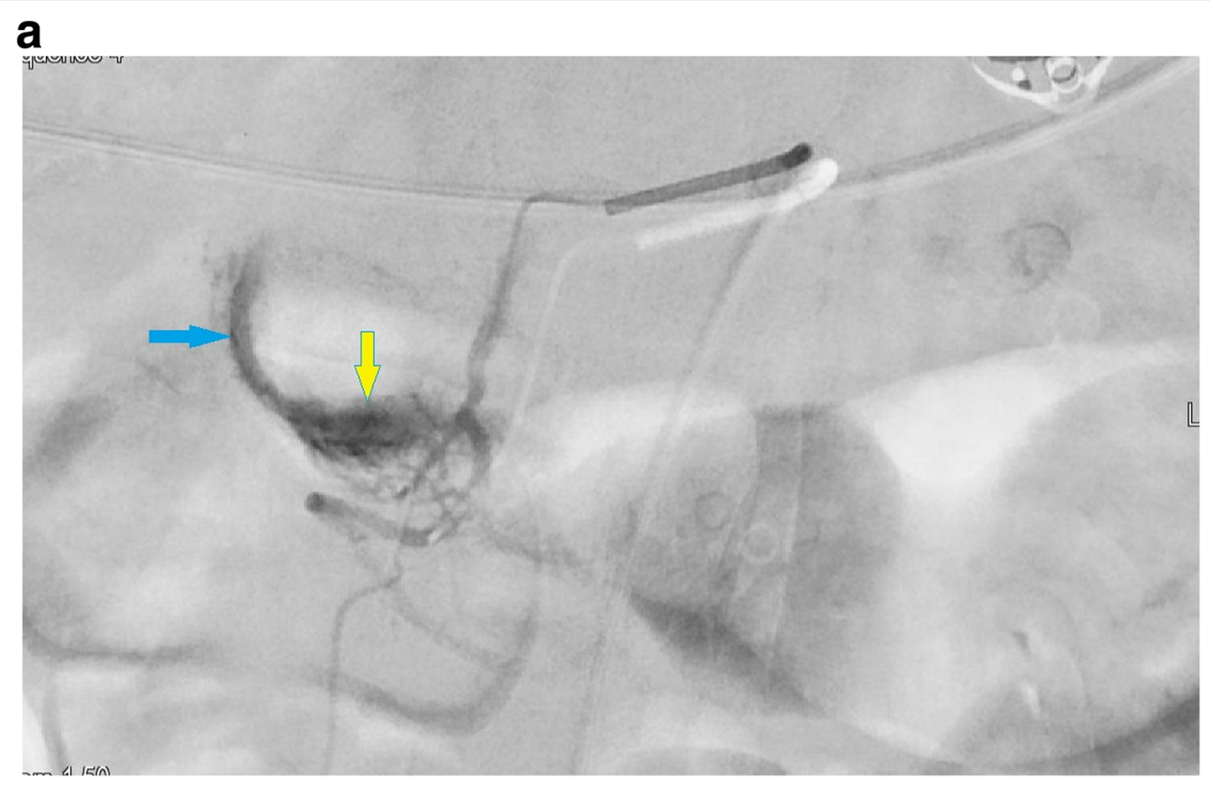

b

C
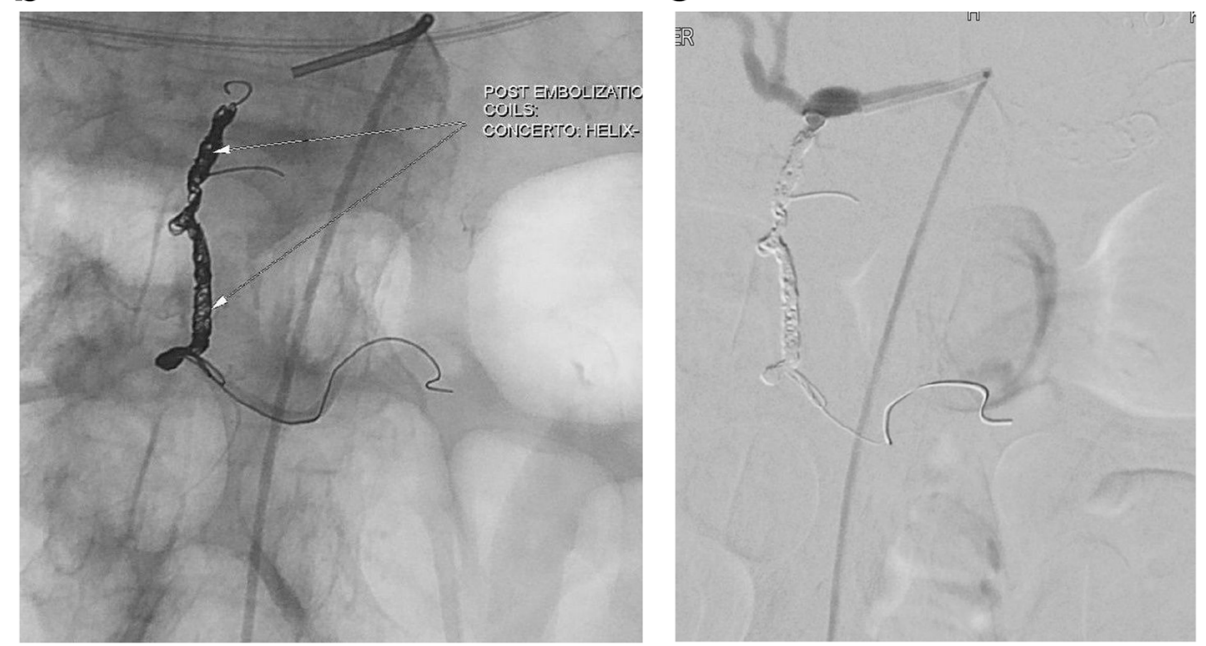

Fig. 2 a-c Celiac arteriography a angiodysplasia (yellow arrow) with early venous return (blue arrow), b selective coil embolization, and c postembolization angiogram shows no more abnormal arterial contrast blush

months of clinical follow-up with no further coiling or surgery.

\section{Discussion}

$\mathrm{AD}$ is one of the most recognized causes of the gastrointestinal tract hemorrhage of obscured reason. It is formed of mucosal dilated capillaries that drain into the submucosal tortuous vein. Mostly, it is less than $5 \mathrm{~mm}$ and can be solitary or multiple. They are more common (about $80 \%$ ) to occur in the proximal part of the colon while the small bowel and upper GIT are of less incidence [1-4].

Clinical picture is variable, and the patient can be asymptomatic, having iron deficiency anemia (due to frequent bleeding) or even can be presented by acute GIT bleeding. The lesions can be undetectable at barium study and endoscopy as well as not seen at laparotomy $[3,8]$.

Arteriography can play an essential role in the diagnosis of angiodysplasia with high-sensitivity and low-falsenegative results $(<12 \%)$ when using selective angiography technique. It can show the feeding arteriole and the related localized, berry-like vascular tufts with early filling of efferent engorged veins $[3,10,11]$.

While the etiology of duodenal angiodysplasia is still not clear, some theories were postulated to explain its pathogenesis in the view of its similarity with colonic angiodysplasia. Some considered angiodysplasia to be a congenital process in patients under 20 years old with increased its incidence as the age advances which may attributed to a 
degenerative process. On the other hand, some considered it as an acquired lesion, attributing their assumption to the higher prevalence in old patients (> 60 years) in most studies [3,9].

The relation of angiodysplasia to renal failure was extensively studied. It showed a higher prevalence of these lesions in renal failure patients. Some authors assumed that it can be bleeding in up to $32 \%$ of cases associated with renal failure and rebleeding in $25-47 \%$ of cases. This can be explained by several reasons such as the platelet and use of anti-coagulants in renal patient. They recommended to consider angiodysplasia as an important reason in the differential diagnosis of GIT bleeding in chronic renal failure $[1,3,9,12,13]$.

The minimal invasiveness and availability of $\mathrm{CT}$ in the emergency situations together with the technical advancement of multidetector CT (MDCT) especially its high-temporal resolution made it feasible to have highresolution multiphasic 3D datasets in short acquisition times, thus raised the usage of CT angiography in vascular lesions especially in the scenario of active hemorrhage. This can identify the bleeding source as active extravasation of the contrast material with high sensitivity and accuracy. The accuracy of CT angiography in finding the reason of GI hemorrhage can be more than $80 \%$. Angiodysplasia is typically appear as a vascular tuft and a large draining vein $[4,14-20]$.

The management of $\mathrm{AD}$ is highly dependable on the clinical situation. For incidentally discovered asymptomatic non-bleeding $\mathrm{AD}$, no intervention may be required as the risk of future bleeding is low. While treatment should be considered if no other source of occult or overt GIT bleeding could be identified [1].

Superselective transcatheter embolization is currently the method of choice for managing bleeding AD especially in patients with failed endoscopic therapy or unsuitable for endoscopy and also as an alternative to surgery in high-risk patients. It shows success rate of up to $90 \%$. It usually uses biodegradable gelatin sponges and microcoils with the possibility for re-embolization for rebleeding cases [1, 21-25]. The complication of that procedure can include hematomas, dissection of artery, thrombus, pseudo-aneurysm, and bowel necrosis. However, the overall complications can be about $5-9 \%$ with less than $2 \%$ to be serious ones in recent techniques [1,23-28].

In the current case, coil embolization was done through super-selective catheterization of the feeding vessel with successful outcome and no acute complication occurred.

\section{Conclusion}

$\mathrm{AD}$ is a rare but important cause that should be considered in the differential diagnosis of GIT bleeding especially in the older patients. It should be looked for in CT angiography done in such clinical situation. Superselective coil embolization is a safe and effective technique to manage bowel AD.

\section{Abbreviations \\ GIT: Gastro-intestinal tract; AD: Angiodysplasia; DSA: Digital subtraction angiography; CT: Computed tomography; SMA: Superior mesenteric artery; MDCT: Multidetector CT \\ Acknowledgements \\ Not applicable. \\ Authors' contributions \\ AT: conceived of the study, participated in its design and coordination, drafted the manuscript, and carried out radiological results. Wl: participated in study design and carried out interventional procedure. AM: participated in study design and helped in drafting the manuscript and radiological results. EM: participated in study design and helped in drafting the manuscript and radiological results. The authors read and approved the final manuscript.}

\section{Funding}

Not applicable.

\section{Availability of data and materials}

The data that support the findings of this study are available on request from the corresponding author.

\section{Ethics approval and consent to participate}

This study was approved from ethical committee of the institution as well as informed written consent from the patient included in this study was taken.

\section{Consent for publication}

All authors read and approved the final manuscript.

Patient included in this research gave written informed consent to publish the data contained within this study.

\section{Competing interests}

The authors declare that they have no competing interests.

\section{Author details}

${ }^{1}$ Department of Radio-diagnosis, Faculty of Medicine, Zagazig University, PO BOX 184, Sharkia, Zagazig 44511, Egypt. 'Radio-Diagnosis Department, King Hamad University Hospital Bahrain, Al Sayh, Bahrain. ${ }^{3}$ Department of Radio-Diagnosis, King Hamad University Hospital Bahrain, Al Sayh, Bahrain. ${ }^{4}$ Department of Radio-Diagnosis, Faculty of Medicine, Zagazig University, Zagazig, Egypt.

Received: 17 August 2020 Accepted: 24 January 2021

Published online: 02 February 2021

\section{References}

1. Sami SS, Araji SA, Ragunath K (2014) Review article: gastrointestinal angiodysplasia - pathogenesis, diagnosis and management. Aliment Pharmacol Ther 39:15-34

2. Athanasoulis CA, Galdabini JJ, Waltman AC, Novelline RA, Greenfield AJ, Ezpeleta ML (1977) Angiodysplasia of the colon: a cause of rectal bleeding. Cardiovasc Radiol 1:3-13

3. Coral RP, Mastalir FP, Mastalir ET (2007) Duodenal angiodysplasia: case report and literature review. ABCD Arq Bras Cir Dig 20(2):127-129

4. Artigas JM, Martí M, Soto JA, Esteban H, Pinilla I, Guillén E, Multidetector CT (2013) Angiography for Acute Gastrointestinal Bleeding: Technique and Findings. RadioGraphics 33:1453-1470

5. Kerr SF, Puppala S (2011) Acute gastrointestinal haemorrhage: the role of the radiologist. Postgrad Med J 87(1027):362-368

6. Friebe B, Wieners $G$ (2011) Radiographic techniques for localization and treatment of gastrointestinal bleeding of obscure origin. Eur J Trauma Emerg Surg 37(4):353-363

7. Kuo WT (2004) Transcatheter treatment for lower gastrointestinal hemorrhage. Tech Vasc Interv Radiol 7:143-150 
8. Gunsar F, Yildiz C, Aydin A, Ozutemiz OA (2001) Angiodysplasia in a duodenal diverticulum as an unusual cause of upper gastrointestinal bleeding. Eur J Gastroenterol Hepatol 13:717-719

9. Foutch PG (1993) Angiodysplasia of the gastrointestinal tract. Am J Gastroenterol 88:807-818

10. Allison D, Hemingway AP (1981) Angiodysplasia: does old age begin at nineteen? Lancet. 2:979-980

11. Gilmore PR (1988) Angiodysplasia of the upper gastrointestinal tract. J Clin Gastroenterol 10:386-394

12. Boccardo P, Remuzzi G, Galbusera M (2004) Platelet dysfunction in renal failure. Semin Thromb Hemost 30:579-589

13. Kringen MK, Narum S, Lygren I et al (2011) Reduced platelet function and role of drugs in acute gastrointestinal bleeding. Basic Clin Pharmacol Toxicol 108:194-201

14. Johnson JO (2012) Diagnosis of acute gastrointestinal hemorrhage and acute mesenteric ischemia in the era of multi-detector row CT. Radiol Clin North Am 50(1):173-182

15. Frattaroli FM, Casciani E, Spoletini D et al (2009) Prospective study comparing multi-detector row CT and endoscopy in acute gastrointestinal bleeding. World J Surg 33(10):2209-2217

16. Angtuaco TL, Reddy SK, Drapkin S, Harrell LE, Howden CW (2001) The utility of urgent colonoscopy in the evaluation of acute lower gastrointestinal tract bleeding: a 2-year experience from a single center. Am J Gastroenterol 96(6):1782-1785

17. Scheffel H, Pfammatter T, Wildi S, Bauerfeind P, Marincek B, Alkadhi H (2007) Acute gastrointestinal bleeding: detection of source and etiology with multi-detector-row CT. Eur Radiol 17(6):1555-1565

18. Martí M, Artigas JM, Garzón G, Alvarez-Sala R, Soto JA (2012) Acute lower intestinal bleeding: feasibility and diagnostic performance of CT angiography. Radiology 262(1):109-116

19. Martí de Gracia M, Artigas Martín JM (2011) Multidetector computed tomography as the first diagnostic option for acute lower digestive tract bleeding in the emergency department [in Spanish]. Radiologia 53(suppl 1): 43-50

20. Yoon W, Jeong YY, Shin SS et al (2006) Acute massive gastrointestinal bleeding: detection and localization with arterial phase multi-detector row helical CT. Radiology 239(1):160-167

21. Walker TG (2009) Acute gastrointestinal hemorrhage. Tech Vasc Interv Radiol 12:80-91

22. Mirsadraee S, Tirukonda P, Nicholson A, Everett SM, MCPherson SJ (2011) Embolization for non-variceal upper gastrointestinal tract haemorrhage: a systematic review. Clin Radiol 66:500-509

23. Funaki B (2002) Endovascular intervention for the treatment of acute arterial gastrointestinal hemorrhage. Gastroenterol Clin North Am 31:701-713

24. Kuo WT, Lee DE, Saad WEA, Patel N, Sahler LG, Waldman DL (2003) Superselective microcoil embolization for the treatment of lower gastrointestinal hemorrhage. J Vasc Interv Radiol 14:1503-1509

25. Bandi R, Shetty PC, Sharma RP, Burke TH, Burke MW, Kastan D (2001) Superselective arterial embolization for the treatment of lower gastrointestinal hemorrhage. J Vasc Interv Radiol 12:1399-1405

26. Patel TH, Cordts PR, Abcarian P, Sawyer MA (2001) Will transcatheter embolotherapy replace surgery in the treatment of gastrointestinal bleeding? Curr Surg 58:323-327

27. Schenker MP, Duszak R Jr, Soulen MC et al (2001) Upper gastrointestinal hemorrhage and transcatheter embolotherapy: clinical and technical factors impacting success and survival. J Vasc Interv Radiol 12:1263-1271

28. Xu M, Zhu X, Liu Y et al (2018) Embolization of arterial gastrointestinal hemorrhage with Fuaile medical adhesive. J Chin Med Assoc 81:636-642

\section{Publisher's Note}

Springer Nature remains neutral with regard to jurisdictional claims in published maps and institutional affiliations.

\section{Submit your manuscript to a SpringerOpen ${ }^{\circ}$ journal and benefit from:}

- Convenient online submission

- Rigorous peer review

- Open access: articles freely available online

- High visibility within the field

- Retaining the copyright to your article

Submit your next manuscript at $\boldsymbol{\nabla}$ springeropen.com 